\title{
Informing adaptive management strategies: Evaluating a mechanism to predict the likely qualitative size of foot-and-mouth disease outbreaks in New Zealand using data available in the early response phase of simulated outbreaks
}

\author{
Robert Sanson ${ }^{1}$, Zhidong $\mathrm{Yu}^{2}$, Thomas Rawdon ${ }^{3}$, and Mary van Andel $^{2}$ \\ ${ }^{1}$ AsureQuality New Zealand \\ ${ }^{2}$ New Zealand Ministry for Primary Industries \\ ${ }^{3}$ Ministry for Primary Industries
}

June 22, 2020

\begin{abstract}
The objective of the study was to define and then evaluate an early decision indicator (EDI) trigger that operated within the first 5 weeks of a response that would indicate a large outbreak of FMD was developing, in order to be able to inform control options within an adaptive management framework. To define the trigger, a previous dataset of 10,000 simulated FMD outbreaks in New Zealand, controlled by the standard stamping-out approach, was re-analysed at various time points between days 11-35 of each response. The two predictive metrics adopted comprised the mean third quartiles of cumulative numbers of infected premises (IPs) at weekly time points, and estimated dissemination rate (EDR) values indicating sustained spread, specifically $>2.0$ between days $11-14$ or $>1.5$ at any time point between days $15-35$ of the response. To evaluate the trigger, the trigger was parameterized within the InterSpread Plus modelling framework, and a new series of simulation generated. The trigger was treated like a series of diagnostic tests that were applied during days 11-35 of each simulated outbreak, and its results recorded and then compared to the final size of each outbreak. The performance of the test was then evaluated across the population of outbreaks, and the sensitivity (Se), specificity (Sp), positive predictive value (PPV) and negative predictive value (NPV) calculated. The Se, Sp, PPV and NPV for predicting large outbreaks were 0.997, 0.513, 0.404 and 0.998 respectively. The study showed that the complex EDI incorporating both the cumulative number of IPs and EDR was very sensitive to detecting large outbreaks, although not all outbreaks predicted to be large were so, whereas outbreaks predicted to be small invariably were small. Therefore, it shows promise as a tool that could support an adaptive management approach to FMD control.
\end{abstract}

\section{Title}

Informing adaptive management strategies: Evaluating a mechanism to predict the likely qualitative size of foot-and-mouth disease outbreaks in New Zealand using data available in the early response phase of simulated outbreaks

\section{Authors}

Robert L. Sanson ${ }^{1}$, Zhidong D. Yu ${ }^{2}$, Thomas G. Rawdon ${ }^{3}$, Mary van Andel ${ }^{4}$

${ }^{1}$ AsureQuality Limited, Palmerston North, New Zealand

${ }^{2}$ Food Science and Risk Assessment, Ministry for Primary Industries, Wellington, New Zealand

${ }^{3}$ Diagnostics and Surveillance Services Directorate, Ministry for Primary Industries, Upper Hutt, New Zealand 
${ }^{4}$ Office of the Chief Departmental Scientist, Ministry for Primary Industries, Wellington, New Zealand

\section{Summary}

The objective of the study was to define and then evaluate an early decision indicator (EDI) trigger that operated within the first 5 weeks of a response that would indicate a large outbreak of FMD was developing, in order to be able to inform control options within an adaptive management framework. To define the trigger, a previous dataset of 10,000 simulated FMD outbreaks in New Zealand, controlled by the standard stamping-out approach, was re-analysed at various time points between days 11-35 of each response. The two predictive metrics adopted comprised the mean third quartiles of cumulative numbers of infected premises (IPs) at weekly time points, and estimated dissemination rate (EDR) values indicating sustained spread, specifically $>2.0$ between days 11-14 or $>1.5$ at any time point between days 15-35 of the response. To evaluate the trigger, the trigger was parameterized within the InterSpread Plus modelling framework, and a new series of simulation generated. The trigger was treated like a series of diagnostic tests that were applied during days 11-35 of each simulated outbreak, and its results recorded and then compared to the final size of each outbreak. The performance of the test was then evaluated across the population of outbreaks, and the sensitivity (Se), specificity (Sp), positive predictive value (PPV) and negative predictive value (NPV) calculated. The Se, Sp, PPV and NPV for predicting large outbreaks were 0.997, 0.513, 0.404 and 0.998 respectively. The study showed that the complex EDI incorporating both the cumulative number of IPs and EDR was very sensitive to detecting large outbreaks, although not all outbreaks predicted to be large were so, whereas outbreaks predicted to be small invariably were small. Therefore, it shows promise as a tool that could support an adaptive management approach to FMD control.

\section{Key Words}

adaptive management strategies; early decision indicators; foot-and-mouth disease; computer simulation

$$
\begin{aligned}
& \text { Abbreviations } \\
& \text { EDI - early decision indicators } \\
& \text { EDR - estimated dissemination rate } \\
& \text { FMD - foot-and-mouth disease } \\
& \text { IP - infected premises } \\
& \text { ISP - InterSpread Plus } \\
& \text { NPV - negative predictive value } \\
& \text { PPV - positive predictive value } \\
& \text { Se - sensitivity } \\
& \text { Sp - specificity }
\end{aligned}
$$

\section{Ethics Statement}

The authors confirm that the ethical policies of the journal, as noted on the journal's author guidelines page, have been adhered to. No ethical approval was required as this article reports on a computer simulation study.

\section{Introduction}

New Zealand has had a simulation model of foot-and-mouth disease (FMD) built within the InterSpread Plus (ISP) simulation system since 1993 (Sanson, 1993; Owen et al. 2011; Stevenson et al. 2013). This model has been periodically improved and refined, both in terms of the capability of the simulation software and in the parameters that represent the epidemiology of the disease and the behaviour of the livestock sectors 
and has been used to evaluate a range of response options should New Zealand ever experience an outbreak (see for example Sanson et al. 2017).

There is international interest in being able to predict the size and /or duration of outbreaks of FMD using variables or measures available or calculable from data captured early in the response (Tomassenet al. 2002; Hutber et al. 2006; Halasa et al.2013; Sarandopoulos, 2015; Garner et al. 2016).

One of these measures to predict outbreak trend is the estimated dissemination rate (EDR). The dissemination rate of a disease is the average number of herds or premises to which a disease agent is delivered by each infected herd. In practice it is hard to measure, however, Miller (1979) proposed an EDR for FMD as the ratio of cumulative incidence in one week to cumulative incidence in the previous week, based on the premise that the herds detected in a given week were likely infected by herds detected the previous week. Thus EDR provides a simple way to assess the observed rate of disease spread and gain insight as to whether an epidemic is likely to continue (EDR $>1$ ) or whether it is being brought under control $(\mathrm{EDR}<1)$. The time period is based around the inter-farm generation time period for the disease in question, which in the case of FMD is approximately 4-7 days (Hugh-Jones \& Tinline, 1976).

These measures have collectively been referred to as early decision indicators (EDIs) (Garner et al. 2016). The benefit of knowing whether an outbreak is likely to be large or small early in the outbreak is that this knowledge can help guide the control and eradication strategy decision making via an adaptive management approach (Geet al. 2010; Halasa et al. 2013; Shae et al. 2014). This may include if vaccination should be adopted as an additional response measure. Various studies have indicated that emergency vaccination, if implemented early for large FMD outbreaks, benefit earlier control (Sanson et al. 2014; Sanson et al. 2017; Rawdon et al. 2018). Vaccination, however, showed little positive effect to control small outbreaks (Dürr et al. 2014), and it could lead to unnecessary competition for resources which are already strained and, a new complex task to monitor and manage vaccinated animals post outbreak for regaining FMD-free status and resuming international trade.

In the study by Garner and colleagues (2016), FMD modelling teams in Australia and New Zealand each generated 10,000 outbreaks of FMD in their respective countries using AusSpread (Garner \& Beckett, 2005) and ISP respectively. Linear regression, classification and regression tree, and boosted regression tree analyses were used to quantify the predictive value of a set of parameters on three outcome variables of interest: the total number of IPs, outbreak duration, and the final area under control (AUC). The number of IPs, number of pending culls, AUC, EDR, and cattle density around the index farm at days 7, 14, and 21 following first detection were statistically associated with each of the outcome variables.

ISP supports the definition of trigger points that can be used to invoke specific actions that affect disease transmission or control. These include measures such as the numbers of detected IPs or EDR exceeding specified values by certain time points relative to the start of the simulation or first detection. Given the predictive ability of IP numbers and EDR values early in the response as reported by Garner and co-workers (2016), this study re-analysed the New Zealand dataset at additional time points to define a time-varying complex EDI trigger that could be specified within the ISP environment and triggered in 'real-time' during further simulated outbreaks. In a sense, this trigger would behave as a diagnostic test applied to each outbreak during the early stages of the outbreak, and therefore its diagnostic performance could be assessed prospectively by comparing the test results early in the response to the final sizes and durations. This paper reports on the performance of this trigger within a larger study exploring the benefits of emergency vaccination to augment $\mathrm{SO}$ within an adaptive management framework.

\section{Methods}

Part One of the study re-analysed the dataset of 10,000 simulated outbreaks of FMD in New Zealand generated during the study by Garner and colleagues (2016), to calculate the third quartile values for the numbers of IPs and the observed EDR values at days 14, 21, 28 and 35 of the response (post first-detection). The purpose was to use these values to define a time-varying series of triggers that operated within specified time periods within the ISP modelling platform. These time periods were defined as response days (post first 
detection) 11-14, 15-21, 22-28 and 29-35 inclusive.

In Part Two of the study, the threshold values for the numbers of IPs and EDR for each time period were specified as a complex EDI trigger within the ISP platform, and the New Zealand Standard Model of FMD was initialized to simulate a further set of FMD incursions into New Zealand. The underlying farm denominator dataset was based on a September 2015 extract of AgriBase (Sanson 2005), a national farms database in New Zealand, owned and operated by AsureQuality Limited, a state-owned enterprise. The model was set up to randomly introduce FMD into farms in the upper North Island, within an area termed the "Auckland Mega-region", which was created by combining the Northland, Auckland, Waikato and Bay of Plenty regions.

Before each introduction, several other variables were randomly varied (see Table 1). These included whether the FMD virus was of a type that could be transmitted by the wind, the number of personnel of various roles that were available for response duties, the number of direct and/or indirect contacts that a tracer could process per shift, and the number of farms that a surveillance veterinarian could visit per day. Once detected by passive surveillance, each outbreak was controlled by standard stamping-out (SO) measures, including tracing of movements, quarantine and depopulation of IPs, movement controls and active surveillance by patrol veterinarians. Each simulated outbreak continued until eradication or to a maximum of 365 days if not eradicated.

Data generated during each simulated outbreak was stored in a Sqlite3 database. The main outputs of the model for each iteration were whether the EDI trigger fired and if so when, the number of farms infected each day, the number of IPs detected each day, the number of farms depopulated per day, and the number of personnel used in response duties per day by role type. From these, further outputs were derived, including the farm type of the primary case, the day of first detection, the total number of IPs detected, and the duration of each outbreak (day of last detection - day of first detection +1 ). In addition, there were some variables that were able to be measured such as the farm and livestock densities around the primary and index cases (see Table 2).

For the purposes of evaluating the performance of the EDI trigger prospectively, 'large' outbreaks were defined as the final number of IPs being in the upper quartile (i.e. $>75^{\text {th }}$ percentile) of all outbreaks in the Part Two simulations, and 'long' outbreaks were classified as having duration within the upper quartile (> $75^{\text {th }}$ percentile) of epidemic lengths for the Part Two simulations. Performance was evaluated by calculating the sensitivity (the proportion of large / long outbreaks during which the trigger fired [Se]), specificity (the proportion of small / short outbreaks during which the trigger did not fire [Sp]), positive predictive value (the proportion of trigger firings which resulted in large / long outbreaks [PPV]) and negative predictive value (the proportion of outbreaks for which the EDI trigger did not fire which ended up as small / short outbreaks [NPV]) against both IPs and duration using 2x2 contingency tables. Sensitivity analysis of these performance measures was conducted by re-classifying the outbreaks into large or long using the $70^{\text {th }}$ and $80^{\text {th }}$ percentiles.

Statistical analysis included logistic regression of the factors that were associated with the trigger firing, with the independent variables being cattle, sheep, pig and farm densities within a $5 \times 5 \mathrm{~km}$ square centred on the primary case, whether airborne spread could occur or not, the numbers of personnel available by role and the time of first detection. Fitting the model was by backwards, stepwise elimination of non-significant variables $(\mathrm{p}>0.05)$ based on the Wald test. Logistic regression modelling was conducted on the largeand long variables to see if the trigger firing was associated with large or long outbreaks. All analyses were conducted using $\mathrm{R}$ v3.5.3.

\section{Results}

Part One

The third quartile values for the number of IPs detected by Days 14, 21, 28 and 35 for the 10,000 simulated outbreaks were 20,25, 29 and 32 respectively. Based on assessing the EDR values at these same time points 
plus expert opinion, it was decided that 5 -day EDRs $>=2.0$ between days $11-14$ inclusive indicated a large outbreak was brewing, while recording EDR $>=1.5$ between days $15-35$ inclusive also indicated a large outbreak was possible. These values were combined to create the complex EDI as shown in Table 3. If either the number of IPs or the EDR exceeded the stated thresholds during the specified time periods, the trigger fired.

\section{Part Two}

There were 1513 simulated outbreaks generated in Part Two of the study. Of these, there were 1328 outbreaks with $>=2$ detected IPs. Descriptive statistics for these outbreaks are shown in Table 4. There were 114 'extreme' outbreaks that exceeded the size and/or duration of the UK 2001 FMD epidemic, specifically > 2030 IPs or $>221$ days (Anderson Inquiry 2002).

Of the 1328 outbreaks with $>=2$ IPs, the EDI trigger fired 816 times (61.4\% of outbreaks). There were two versions of the EDI trigger, which differed with respect to the EDR component, with one using a 4-day EDR and slightly lower thresholds for triggering than that specified for the 5-day EDR (refer Table 3). Statistical analyses (not reported) indicated there was minimal difference between the overall performance of the two versions, so the results below are based on the combined data.

The day the trigger fired, and whether it was triggered by the cumulative count of IPs exceeding the stated thresholds or EDR are shown in Figure 1. The trigger mostly fired between Days 11 - 21, with the largest proportion of firings on Day 11 (see Figure 1). There were no trigger firings due to IP numbers after Day 21, although there were diminishing numbers of EDR-based trigger firings right through to Day 35.

Table 5 shows the results from the final logistic regression of thetrig variable. The variables catdens, sheepdens andpigdens were all highly collinear with fmdens so were dropped from the model. It can be seen that farm type of the primary case, time to first detection and farm density in the area around the primary case were all associated with the trigger firing. The odds of the trigger firing were higher for grazing / dairy support farms (GRADRY) and pastoral livestock (PLVSTCK) farms relative to dairy farms (DAIRY), whereas lifestyle farms (LIF) were protective (reduced the odds of the trigger firing). There were not enough pig farms selected as the primary case for the role of pig farms to be assessed. Increasing time to first detection and increasing farm density around the primary case were associated with increased odds of the trigger firing.

The third quartiles for the number of IPs and duration amongst the iterations where spread occurred were 49 IPs and 55 days respectively. Iterations that exceeded these values were categorized as large and long outbreaks respectively.

Performance of the EDI trigger was assessed by $2 \times 2$ contingency tables for IPs (Tables 6-8) and durations (Tables 9-11).

Final logistic regression models for variables associated with large and long outbreaks are shown in Tables 12 and 13 respectively. The most influential variable was the trig variable, with the odds of a large or long outbreak being 347 or 33.3 times greater respectively if the trigger fired. Besides this, increasing vet andsurvfpd values reduced the odds of a large outbreak, and increasing $f t$ and survfpd values reduced the odds of a long outbreak.

\section{Discussion}

The time period selected for the operation of the complex EDI trigger was from Days 11 - 35 of each response, with the response beginning once the first IP (index case) was discovered. Day 11 was both the earliest day that a 5-day EDR could be calculated (excluding Day 1 of the response) and the earliest possible date by which a vaccination programme could be initiated, given the requirement to confirm the strain type of FMD virus, receive sufficient vaccine doses from the international vaccine bank and arrange teams of personnel to administer the vaccine. Day 35 represented 5 weeks into the response, and by this time there should be a reasonably clear picture of the spatio-temporal progression of the disease, but still be at an early enough stage that vaccination could assist with outbreak control. 
The thresholds for the numbers of IPs used in the trigger were based on the third quartiles of cumulative IPs detected as at days 14,21, 28 and 35 within the 10,000 outbreaks simulated for the study by Garner and colleagues (2016). Our premise was that should these values be exceeded prior to or equal to those time points, then it would signify a large outbreak was developing (i.e. within the largest $25 \%$ of outbreak sizes). Whilst this was somewhat arbitrary, it was felt that it was not unreasonable.

The trigger mostly fired between Days 11 - 21, with the largest proportion of firings on Day 11 (see Figure 1). Overall, the trigger had very high sensitivities for predicting large and long epidemics. Conversely, the trigger had poor specificity and PPV, but very high NPV. What this means is that the trigger missed very few outbreaks that subsequently turned out to be large, but not all of the outbreaks predicted to be large or long turned out to be so. Putting it another way, if the trigger did not fire during Days 11 - 35, the subsequent outbreak size was most likely to be small or short. Sensitivity analysis of the thresholds for classifying outbreaks as large or long showed that the performance characteristics of the trigger did not change much, with slight increases in sensitivities as the threshold was raised from the $70^{\text {th }}$ percentile to the $80^{\text {th }}$ percentile, with corresponding small decreases in specificities. The ability to identify most of the large and long epidemics during the early stage of simulated responses showed the utility of the trigger in providing inputs that could assist the decision-makers responsible for making response decisions based on epidemic situation, including the need for urgent implementation of vaccination to strengthen the eradication efforts.

An assessment of the relative performances of the IP and EDR components showed that they were both of similar high sensitivity, but the EDR component had lower specificity and PPV measures (see Tables 8 \& 11). Note that the IP and EDR components were not strictly independent of each other, as they operated in parallel and the threshold that fired first was the one that was recorded. Further, the trigger could only fire once for each outbreak, so this did not mean that thresholds were not exceeded later, it's just that there were relatively few first firings beyond Day 21. It appears that the lower specificities and PPVs demonstrated by the EDR component were because the 5-day EDR values were less stable and could exhibit short-term spikes. Nevertheless, the overall results suggest that the complex EDI used could identify large and long outbreaks during the early stages of simulated outbreaks.

There are several options for trying to improve the overall performance of the EDI trigger. Given the high number of firings that occurred on the first day that the trigger started operating (Day 11), the trigger could be adapted to operate from Day 8 onwards by using a 4-day EDR together with cumulative IP number thresholds appropriate to the time frame, then switch to a 5-day EDR on Day 10 and then to a 6-day EDR on Day 12 of the response. It must be acknowledged however, that it is very difficult to get accurate assessments of disease dynamics very early in the response phase, as new IPs would comprise a combination of cases that were infected during the 'Silent Phase' but still be in the process of being discovered, as well as newly infected cases. To try and improve the specificity of the trigger, particularly of the EDR component, longer baseline time periods for the EDR calculations would reduce the likelihood of short-term spikes that are not truly indicative of sustained transmission. Further, EDR thresholds could be raised slightly, or it could be made a requirement that the EDR thresholds had to be exceeded on two separate occasions for the trigger to fire.

Extrinsic factors that influenced the trigger firing were farm type of the primary case, time to first detection and farm density in the vicinity of the primary case. Time to first detection has been associated with larger outbreaks as reported by other researchers (McLaws \& Ribble, 2007; East et al. 2015). Type of primary case farm would affect movement frequencies, and increasing farm densities would allow for higher levels of local spread.

The two logistic regression models that explored factors associated with large and long outbreaks (see Tables 12 and 13 respectively) showed that trigger firing was by far the most influential factor: the odds of a large or long outbreak were many times higher when the trigger fired. The other significant factors were related to resourcing issues - affecting active surveillance and depopulation capabilities. Veterinarians (vet variable) have roles in surveillance within the $3 \mathrm{~km}$ patrol zones around IPs and in traced farms as well as in depopulation, and Field Technicians ( $f t$ variable) have a role in depopulation. The survfpd variable indicates 
the number of farms that a veterinarian can visit per day, so increasing numbers of farms that could be visited per day by veterinarians had an effect of reducing the odds of a large and long outbreak.

In conclusion, the study showed that an EDI using a combination of cumulative IP numbers and EDR values indicating sustained spread are highly predictive of the eventual size of the outbreak. Further evaluation and improvement of the complex EDI may lead to a valuable tool to predict the eventual epidemic size based on the observed IP numbers and rates of spread during the early stages of outbreaks. Linking temporal changes in observed dissemination rates to the implementation of response measures can provide insights into the effectiveness of the controls already in place and, if required, highlight the need for additional response measures to deliver more effective control of an FMD outbreak.

\section{Acknowledgements}

The research was conducted as part of Biosecurity Readiness project funded by the New Zealand Ministry for Primary Industries (MPI). MPI staff provided technical input and oversight to ensure the development and delivery of what is required to support MPI readiness work. AsureQuality Limited is thanked for providing access to its AgriBase farm database.

\section{Conflict of interest statement}

To the authors' best knowledge, there are no conflicts of interest to report.

\section{Data Availability Statement}

The data that support the findings of this study are available from the corresponding author upon reasonable request.

\section{References}

Anderson Inquiry (2002). Foot-and-mouth disease 2001: Lessons Learned Inquiry , pp 169. The Stationery Office, London. https://webarchive.nationalarchives.gov.uk/20100809105008/http://archive.cabinetoffice.gov.uk/fmd/fmd_report/report/index.htm (accessed 26 November 2019)

Dürr, S., Fasel-Clemenz, C., Thür, B., Schwermer, H., Doherr, M. G., zu Dohna, H., Carpenter, T.E., Perler, L., \& Hadorn, D.C. (2014). Evaluation of the benefit of emergency vaccination in a foot-and-mouth disease free country with low livestock density. Preventive Veterinary Medicine 113, 34-46.

East, I. J., Martin, P. A. J., Langstaff, I., Wicks, R. M., Sergeant, E. S. G., \& Garner, M. G. (2015). Assessing the delay to detection and the size of the outbreak at the time of detection of incursions of foot and mouth disease in Australia. Preventive Veterinary Medicine 123, 1-11. doi:10.1016/j.prevetmed.2015.12.005

Garner, M. G., \& Beckett, S. D. (2005). Modelling the spread of foot-and-mouth disease in Australia. Australian Veterinary Journal 83(12), 758-766.

Garner, M. G., East, I. J., Stevenson, M. A., Sanson, R. L., Rawdon, T. G., Bradhurst, R. A., Roche, S. E., Van Ha, P., \& Kompas, T. (2016). Early Decision Indicators for Foot-and-Mouth Disease Outbreaks in Non-Endemic Countries. Frontiers in Veterinary Science 3:109. http://dx.doi.org/10.3389/fvets.2016.00109

Ge, L., Mourits, M. C. M., Kristensen, A. R., \& Huirne, R. B. M. (2010). A modelling approach to support dynamic decision-making in the control of FMD epidemics. Preventive Veterinary Medicine 95, 167-174.

Halasa, T., Willeberg, P., Christiansen, L. E., Boklund, A., Alkhamis, M., Perez, A., \& Enøe, C. (2013). Decisions on control of foot-and-mouth disease informed using model predictions.Preventive Veterinary Medicine 112, 194-202. http://dx.doi.org/10.1016/j.prevetmed.2013.09.003

Hugh-Jones, M.E., \& Tinline, R.R. (1976). Studies on the 1967-68 foot and mouth disease epidemic: incubation period and the herd serial interval. Journal of Hygiene, Cambridge, 77, 141-153. 
Hutber, A., Kitching, P., \& Pilipcinec, E. (2006). Predictions for the timing and use of culling or vaccination during a foot-and-mouth disease epidemic. Research in Veterinary Science 81:31-36. doi:10.1016/j.rvsc.2005.09.014

McLaws, M., \& Ribble, C. (2007). Description of recent foot and mouth disease outbreaks in nonendemic areas: exploring the relationship between early detection and epidemic size. Canadian Veterinary Journal 48:1051-1062.

Miller, W. M. (1979). A state-transition model of epidemic foot-and-mouth disease. In: E.H. McCauley, J.C. New, N.A. Aulaqi, W.B. Sundquist \& W.M. Miller, A Study of the Potential Economic Impact of Foot-and-mouth Disease in the United States, pp 113-131. University of Minnesota, St. Paul, Minnesota.

Owen, K., Stevenson, M. A., \& Sanson, R.L. (2011). A sensitivity analysis of the New Zealand standard model of foot-and-mouth disease.Revue Scientifique et Technique de l'Office International des Epizooties 30(2), 513-526.

Rawdon, T. G., Garner, M. G., Sanson, R. L., Stevenson, M. A , Cook, C., Birch, C., Roche, S. E., Patyk, K. A., Forde-Folle, K. N., Dubé, C., Smylie, T., \& Yu, Z. D. (2018). Evaluating vaccination strategies to control foot-and-mouth disease: a country comparison study.Epidemiology and Infection 146, 1138-1150. https://doi.org/10.1017/S0950268818001243

Roche, S. E., Garner, M. G., Sanson, R. L., Cook, C., Birch, C., Backer, J. A., Dubé, C., Patyk, K. A., Stevenson, M. A., Yu, Z., Rawdon, T. G., \& Gauntlett, F. (2015). Evaluating vaccination strategies to control foot-and-mouth disease: a model comparison study. Epidemiology $\&$ Infection 143 (6), 1256-1275. http://dx.doi.org/10.1017/S0950268814001927

Sanson, R. L. (1993). The development of a decision support system for an animal disease emergency . PhD thesis, Massey University, Palmerston North.

Sanson, R. L. (2005). The AgriBase ${ }^{\mathrm{TM}}$ farm location database. Proceedings of the New Zealand Society of Animal Production 65, 93-96.

Sanson, R. L., Dubé, C., Cork, S. C., Frederickson, R., \& Morley, C. (2014). Simulation modelling of a hypothetical introduction of foot-and-mouth disease into Alberta.Preventive Veterinary Medicine 114, 151163.

Sanson, R. L., Rawdon, T., Owen, K., Hickey, K., van Andel, M., \& Yu, Z. D. (2017). Evaluating the benefits of vaccination when used in combination with stamping-out measures against hypothetical introductions of foot-and-mouth disease into New Zealand: a simulation study. New Zealand Veterinary Journal 65(3), 124133. http://dx.doi.org/10.1080/00480169.2016.1263165

Sarandopoulos, J. (2015). Early Predictors of the Size and Duration of Foot-and-Mouth Disease Epidemics [Unpublished Bachelor of Science Honours Thesis]. Melbourne, Australia: Faculty of Veterinary and Agricultural Sciences, University of Melbourne.

Shea, K., Tildesley, M. J., Runge, M. C., Fonnesbeck, C. J., \& Ferrari, M. J. (2014). Adaptive management and the Value of Information: Learning Via Intervention in Epidemiology. PLoS Biol 12(10), e1001970. http://dx.doi.org/10.1371/journal.pbio.1001970

Stevenson, M. A., Sanson, R.L., Stern, M. W., O'Leary, B. D., Sujau, M., Moles-Benfell, N., \& Morris, R. S. (2013). InterSpread Plus: a spatial and stochastic simulation model of disease in animal populations.Preventive Veterinary Medicine 109, 10-24. http://dx.doi.org/10.1016/j.prevetmed.2012.08.015

Tomassen, F., de Koejier, A., Mourits, M., Dekker, A., Bouma, A., \& Huirne, R. (2002). A decision-tree to optimise control measures during the early stage of a foot-and-mouth disease epidemic. Preventive Veterinary Medicine 54, 301-324. doi:10.1016/S0167-5877(02)00053-3

Tables 
Table 1. Parameters randomly set prior to each simulated outbreak.

\begin{tabular}{|c|c|}
\hline Variable & Description and Range of Values \\
\hline admin & $\begin{array}{l}\text { The number of ADMIN personnel available (range } \\
7-995 \text { for the first } 5000 \text { iterations, } 10-200 \text { for the } \\
\text { next } 1000 \text { iterations) }\end{array}$ \\
\hline$f t$ & $\begin{array}{l}\text { The number of FT personnel available (range } \\
7-720 \text { for the first } 5000 \text { iterations, } 10-200 \text { for the } \\
\text { next } 1000 \text { iterations) }\end{array}$ \\
\hline vet & $\begin{array}{l}\text { The number of VET personnel available (range } \\
7-995 \text { for the first } 5000 \text { iterations, } 10-200 \text { for the } \\
\text { next } 1000 \text { iterations) }\end{array}$ \\
\hline arm_slgt & $\begin{array}{l}\text { The number of ARM \& SLGT personnel available } \\
\text { (7-140 for all } 6000 \text { iterations) }\end{array}$ \\
\hline anhandler & $\begin{array}{l}\text { The number of ANHANDLER personnel available } \\
\text { (7-200 for all } 6000 \text { iterations) }\end{array}$ \\
\hline strategy & $\begin{array}{l}\mathrm{SO}=\text { Stamping out only; TRV }=\mathrm{SO}+\text { Trigger } \\
\text { Vaccination if the EDI trigger fired (refer Table } 1) \text {; }\end{array}$ \\
\hline air & Airborne spread; $1=$ on; $0=$ off \\
\hline tracespsh & Traces per shift completed by each tracer $(10-30)$ \\
\hline survfpd & Farms visited by surveillance vets per day $(2-6)$ \\
\hline
\end{tabular}

Table 2. Variables derived or calculated during the simulations.

\begin{tabular}{ll}
\hline Variable & Description and Range of Values \\
\hline firstdet & The time in days from the start of the simulation to the first detection \\
primclass & The farm class of the primary case in each iteration \\
fmdens & The farm density (farms $\left./ \mathrm{km}^{2}\right)$ calculated from a $5 \times 5 \mathrm{~km}$ square centred on the primary case \\
catdens & The cattle density $\left(\right.$ cattle $\left./ \mathrm{km}^{2}\right)$ calculated from a $5 \times 5 \mathrm{~km}$ square centred on the primary case \\
sheepdens & The sheep density $\left(\right.$ sheep $\left./ \mathrm{km}^{2}\right)$ calculated from a $5 \times 5 \mathrm{~km}$ square centred on the primary case \\
pigdens & The pig density (pigs $\left./ \mathrm{km}^{2}\right)$ calculated from a $5 \times \mathrm{km}$ square centred on the primary case \\
triggerday & The day that the EDI trigger fired, otherwise 999 \\
dets & The number of detected farms $(\mathrm{IPs})$ \\
epil & The duration of the response (time from first to last detected case in days) \\
trig & A binary variable indicating whether the EDI trigger fired $(0=$ no; $1=$ yes $)$ \\
large & A binary variable indicating whether the number of IPs was in the upper quartile for the number of IPs $(0=\mathrm{r}$ \\
long & A binary variable indicating whether the epidemic duration was in the upper quartile for duration $(0=$ no; $1=$ \\
\hline
\end{tabular}

Table 3. Threshold values defined for the early decision indicator (EDI) trigger.

\begin{tabular}{lll}
\hline & Threshold Type & Threshold Type \\
\hline Time period of the response (days) & 5-day EDR & IPs \\
$11-14$ & $>=2.0$ & $>=20$ \\
$15-21$ & $>=1.5$ & $>=25$ \\
$22-28$ & $>=1.5$ & $>=29$ \\
$29-35$ & $>=1.5$ & $>=32$ \\
\hline
\end{tabular}


Table 4. Descriptive statistics for the sizes of outbreaks.

\begin{tabular}{ll}
\hline Description & Statistics \\
\hline No. of iterations & 1513 \\
No. of iterations $>=2$ IPs & 1328 \\
Mean, Median and Range of IPs (all iterations $>=2$ IPs) & $3211,15,2-50,582$ \\
Duration (days) - Mean, Median, Range (all iterations $>=2$ IPs) & $60.7,25,2-360$ \\
No of 'extreme' outbreaks & 114 \\
Mean, Median and Range of IPs ( $>=2$ IPs but excl. extreme outbreaks) & $37.5,12,2-751$ \\
Duration (days) - Mean, Median, Range ( $>=2$ IPs but excl. extreme outbreaks) & $33.8,22,2-217$ \\
\hline
\end{tabular}

Table 5. Final logistic regression model for variables associated with the EDI trigger firing.

\begin{tabular}{llllll}
\hline Variable & OR + & $\mathbf{9 5 \%}$ CI & Std Error & $\mathbf{Z}$ value & P \\
\hline $\begin{array}{l}\text { (Intercept) } \\
\text { primclass }\end{array}$ & 0.07 & $0.041-0.106$ & 0.245 & -11.105 & $<0.0001$ \\
DAIRY & Ref & & & & \\
GRADRY & 1.73 & $1.037-2.892$ & 0.261 & 2.087 & 0.037 \\
LIF & 0.55 & $0.377-0.806$ & 0.193 & -3.067 & 0.002 \\
PIGB & NA & NA & NA & NA & NA \\
PLVSTCK & 1.34 & $0.922-1.939$ & 0.189 & 1.534 & 0.125 \\
firstdet & 1.15 & $1.124-1.185$ & 0.013 & 10.638 & $<0.0001$ \\
fmdens & 1.93 & $1.744-2.153$ & 0.054 & 12.252 & $<0.0001$ \\
\hline
\end{tabular}

+ Odds Ratio (OR) indicates how many times more the trigger firing is likely to be the result for a unit increase in the predictor variable. Values $<1$ are protective.

Table 6 . 2x2 contingency table for the detection of large outbreaks ( $>75^{\text {th }}$ percentile for number of IPs) generated during the Part Two simulations.

\begin{tabular}{llll}
\hline & IPs $>$ 49 $($ large) & IPs $<=49($ small $)$ & Totals \\
\hline Trigger + & 330 & 486 & 816 \\
Trigger - & 1 & 511 & 512 \\
Totals & 331 & 997 & 1328 \\
\hline
\end{tabular}

Table 7. Performance of the EDI trigger for detecting large outbreaks by percentile (95\% Confidence Intervals in brackets).

\begin{tabular}{|c|c|c|c|}
\hline & Threshold for IPs & Threshold for IPs & Threshold for IPs \\
\hline Measure & $\begin{array}{l}70^{\text {th }} \text { percentile }(37 \\
\text { IPs })\end{array}$ & $\begin{array}{l}75^{\text {th }} \text { percentile ( } 49 \\
\text { IPs) }\end{array}$ & $\begin{array}{l}80^{\text {th }} \text { percentile }(71 \\
\text { IPs) }\end{array}$ \\
\hline Sensitivity (Se) & $0.992(0.978-0.997)$ & $0.997(0.983-1)$ & $0.996(0.979-1)$ \\
\hline Specificity (Sp) & $0.547(0.515-0.578)$ & $0.513(0.482-0.543)$ & $0.481(0.451-0.511)$ \\
\hline $\begin{array}{l}\text { Positive Predictive } \\
\text { Value (PPV) }\end{array}$ & $0.483(0.449-0.517)$ & $0.404(0.371-0.438)$ & $0.324(0.292-0.356)$ \\
\hline $\begin{array}{l}\text { Negative Predictive } \\
\text { Value (NPV) }\end{array}$ & $0.994(0.983-0.998)$ & $0.998(0.989-1)$ & $0.998(0.989-1)$ \\
\hline
\end{tabular}


Table 8. Relative performances of the IP-based and EDR-based components of the EDI trigger for predicting large outbreaks ( $>75^{\text {th }}$ percentile of the final number of IPs) with $95 \%$ Confidence Intervals in brackets.

\begin{tabular}{lll}
\hline & EDI Component & EDI Component \\
\hline Measure & IP & EDR \\
Sensitivity (Se) & $0.995(0.973-1)$ & $0.992(0.956-1)$ \\
Specificity (Sp) & $0.862(0.832-0.887)$ & $0.558(0.526-0.59)$ \\
Positive Predictive Value (PPV) & $0.715(0.661-0.764)$ & $0.235(0.201-0.273)$ \\
Negative Predictive Value (NPV) & $0.998(0.989-1)$ & $0.998(0.989-1)$ \\
\hline
\end{tabular}

Table 9 . 2x2 contingency table for detection of long epidemics ( $>75^{\text {th }}$ percentile of duration) generated during the Part Two simulations.

\begin{tabular}{llll}
\hline & $>$ 55 d (long) & $<=55$ d (short) & Totals \\
\hline Trigger + & 321 & 495 & 816 \\
Trigger - & 10 & 502 & 512 \\
Totals & 331 & 997 & 1328 \\
\hline
\end{tabular}

Table 10. Performance of the EDI trigger for detecting long outbreaks by percentile (95\% Confidence Intervals in brackets).

\begin{tabular}{|c|c|c|c|c|}
\hline & & $\begin{array}{l}\text { Threshold for } \\
\text { Duration }\end{array}$ & $\begin{array}{l}\text { Threshold for } \\
\text { Duration }\end{array}$ & $\begin{array}{l}\text { Threshold for } \\
\text { Duration }\end{array}$ \\
\hline Measure & $\begin{array}{l}70^{\text {th }} \text { percentile } \\
\text { (45 days) }\end{array}$ & $\begin{array}{l}70^{\text {th }} \text { percentile } \\
(45 \text { days })\end{array}$ & $\begin{array}{l}75^{\text {th }} \text { percentile } \\
\text { (55 days) }\end{array}$ & $\begin{array}{l}80^{\text {th }} \text { percentile } \\
(70 \text { days })\end{array}$ \\
\hline Sensitivity (Se) & $\begin{array}{l}0.965 \\
(0.942-0.979)\end{array}$ & $\begin{array}{l}0.965 \\
(0.942-0.979)\end{array}$ & $0.97(0.945-0.984)$ & $0.977(0.951-0.99)$ \\
\hline Specificity (Sp) & $\begin{array}{l}0.535 \\
(0.503-0.567)\end{array}$ & $\begin{array}{l}0.535 \\
(0.503-0.567)\end{array}$ & $\begin{array}{l}0.504 \\
(0.473-0.534)\end{array}$ & $\begin{array}{l}0.475 \\
(0.445-0.505)\end{array}$ \\
\hline $\begin{array}{l}\text { Positive } \\
\text { Predictive Value } \\
\text { (PPV) }\end{array}$ & $\begin{array}{l}0.471 \\
(0.437-0.505)\end{array}$ & $\begin{array}{l}0.471 \\
(0.437-0.505)\end{array}$ & $0.393(0.36-0.427)$ & $\begin{array}{l}0.315 \\
(0.284-0.348)\end{array}$ \\
\hline $\begin{array}{l}\text { Negative } \\
\text { Predictive Value } \\
\text { (NPV) }\end{array}$ & $\begin{array}{l}0.973 \\
(0.95509 .984)\end{array}$ & $\begin{array}{l}0.973 \\
(0.95509 .984)\end{array}$ & $0.98(0.964-0.989)$ & $\begin{array}{l}0.988 \\
(0.975-0.995)\end{array}$ \\
\hline
\end{tabular}

Table 11. Relative performance of the IP-based and EDR-based components of the EDI trigger for predicting long outbreaks ( $>75^{\text {th }}$ percentile for duration) with $95 \%$ Confidence Intervals in brackets.

\begin{tabular}{lll}
\hline & EDI Component & EDI Component \\
\hline Measure & IP & EDR \\
Sensitivity (Se) & $0.946(0.903-0.97)$ & $0.936(0.887-0.965)$ \\
Specificity (Sp) & $0.815(0.782-0.844)$ & $0.569(0.536-0.601)$ \\
Positive Predictive Value (PPV) & $0.604(0.547-0.659)$ & $0.278(0.242-0.318)$ \\
Negative Predictive Value (NPV) & $0.98(0.964-0.989)$ & $0.98(0.964-0.989)$ \\
\hline
\end{tabular}


Table 12. Logistic regression model for variables associated with the number of IPs being in the upper quartile.

\begin{tabular}{llllll}
\hline Variable & OR + & $\mathbf{9 5 \%}$ CI & Std Error & $\mathbf{Z}$ value & P \\
\hline (Intercept) & 0.004 & $0.000-0.019$ & 1.0223 & -5.402 & $<0.0001$ \\
vet & 0.9995 & $0.999-1.000$ & 0.0002 & -2.211 & 0.027 \\
survfpd & 0.881 & $0.798-0.972$ & 0.0502 & -2.525 & 0.012 \\
trig & & & & & \\
0 & Ref & & & & \\
1 & 346.97 & $77.900-6105.040$ & 1.0030 & 5.832 & $<0.0001$ \\
\hline
\end{tabular}

+ Odds Ratio (OR) indicates how many times more an upper quartile outbreak is likely to be the result for a unit increase in the predictor variable. Values $<1$ are protective.

Table 13. Logistic regression model for variables associated with the epidemic duration being in the upper quartile.

\begin{tabular}{llllll}
\hline Variable & OR + & $\mathbf{9 5 \%}$ CI & Std Error & $\mathbf{Z}$ value & $\mathbf{P}$ \\
\hline (Intercept) & 0.043 & $0.019-0.087$ & 0.385 & -8.198 & $<0.0001$ \\
ft & 0.9992 & $0.999-1.000$ & 0.0003 & -2.351 & 0.01872 \\
survfpd & 0.88 & $0.797-0.967$ & 0.049 & -2.632 & 0.00848 \\
trig & & & & & \\
0 & Ref & & & & \\
1 & 32.26 & $17.905-65.571$ & 0.328 & 10.602 & $<0.0001$ \\
\hline
\end{tabular}

+ Odds Ratio (OR) indicates how many times more an upper quartile outbreak is likely to be the result for a unit increase in the predictor variable. Values $<1$ are protective.

Figure Legends

Fig 1. Distribution of days when the EDI trigger fired and whether an IP or EDR threshold was exceeded. 


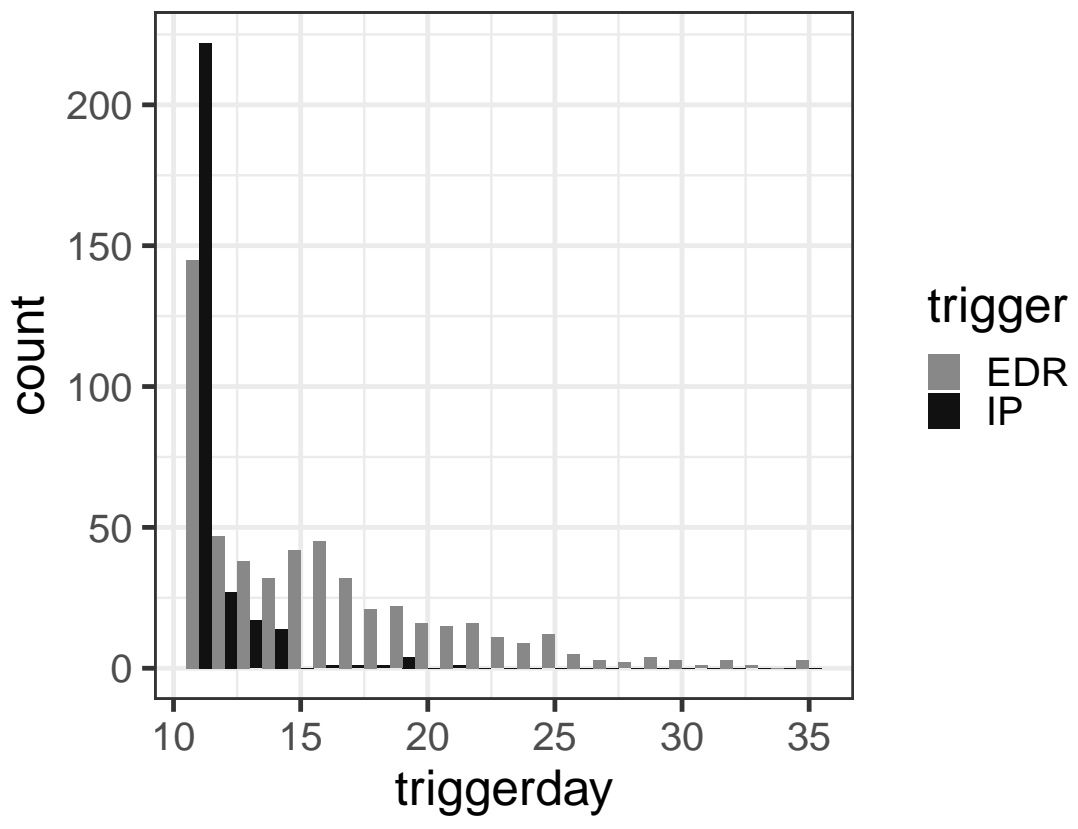

